\title{
Collective Operations on Number-Membered Sets
}

\author{
Artur Korniłowicz \\ Institute of Computer Science \\ University of Białystok \\ Sosnowa 64, 15-887 Białystok \\ Poland
}

\begin{abstract}
Summary. The article starts with definitions of sets of opposite and inverse numbers of a given number membered set. Next, collective addition, subtraction, multiplication and division of two sets are defined. Complex numbers cases and extended real numbers ones are introduced separately and unified for reals. Shortcuts for singletons cases are also defined.
\end{abstract}

MML identifier: MEMBER_1, version: $\underline{7.11 .01 \quad 4.117 .1046}$

The articles [4], [2], [1], and [3] provide the notation and terminology for this paper.

For simplicity, we adopt the following convention: $w, w_{1}, w_{2}$ are elements of $\overline{\mathbb{R}}, c, c_{1}, c_{2}$ are elements of $\mathbb{C}, A, B, C, D$ are complex-membered sets, $F$, $G, H, I$ are extended real-membered sets, $a, b$ are complex numbers, $f, g$ are extended real numbers, $r$ is a real number, and $e$ is a set.

Let us consider $w$. Then $-w$ is an element of $\overline{\mathbb{R}}$. Then $w^{-1}$ is an element of $\overline{\mathbb{R}}$. Let us consider $w_{1}$. Then $w \cdot w_{1}$ is an element of $\overline{\mathbb{R}}$.

Let $a, b, c, d$ be complex numbers. One can check that $\{a, b, c, d\}$ is complexmembered.

Let $a, b, c, d$ be extended real numbers. Observe that $\{a, b, c, d\}$ is extended real-membered.

Let $F$ be an extended real-membered set. The functor $\ominus F$ yielding an extended real-membered set is defined by:

(Def. 1) $\ominus F=\{-w: w \in F\}$. 
Let us note that the functor $\ominus F$ is involutive.

The following propositions are true:

(1) $f \in F$ iff $-f \in \ominus F$.

(2) $-f \in F$ iff $f \in \ominus F$.

Let $F$ be an empty set. One can check that $\ominus F$ is empty.

Let $F$ be an extended real-membered non empty set. Note that $\ominus F$ is non empty.

The following propositions are true:

(3) $F \subseteq G$ iff $\ominus F \subseteq \ominus G$.

(4) If $\ominus F=\ominus G$, then $F=G$.

(5) $\ominus(F \cup G)=\ominus F \cup \ominus G$.

(6) $\ominus(F \cap G)=\ominus F \cap \ominus G$.

(7) $\ominus(F \backslash G)=\ominus F \backslash \ominus G$.

(8) $\ominus(F \dot{-} G)=\ominus F \dot{-} \ominus G$.

(9) $\ominus\{f\}=\{-f\}$.

(10) $\ominus\{f, g\}=\{-f,-g\}$.

Let $A$ be a complex-membered set. The functor $\ominus A$ yields a complex-membered set and is defined as follows:

(Def. 2) $\ominus A=\{-c: c \in A\}$.

Let us note that the functor $\ominus A$ is involutive.

Next we state two propositions:

(11) $a \in A$ iff $-a \in \ominus A$.

(12) $-a \in A$ iff $a \in \ominus A$.

Let $A$ be an empty set. One can check that $\ominus A$ is empty.

Let $A$ be a complex-membered non empty set. One can verify that $\ominus A$ is non empty.

Let $A$ be a real-membered set. One can check that $\ominus A$ is real-membered.

Let $A$ be a rational-membered set. Note that $\ominus A$ is rational-membered.

Let $A$ be an integer-membered set. Observe that $\ominus A$ is integer-membered.

Let $A$ be a real-membered set and let $F$ be an extended real-membered set.

One can verify that $\ominus A$ and $\ominus F$ can be identified when $A=F$.

We now state several propositions:

(13) $A \subseteq B$ iff $\ominus A \subseteq \ominus B$.

(14) If $\ominus A=\ominus B$, then $A=B$.

(15) $\ominus(A \cup B)=\ominus A \cup \ominus B$.

(16) $\ominus(A \cap B)=\ominus A \cap \ominus B$.

(17) $\ominus(A \backslash B)=\ominus A \backslash \ominus B$.

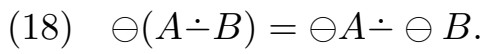


(19) $\ominus\{a\}=\{-a\}$.

(20) $\ominus\{a, b\}=\{-a,-b\}$.

Let $F$ be an extended real-membered set. The functor $F^{-1}$ yields an extended real-membered set and is defined by:

(Def. 3) $F^{-1}=\left\{w^{-1}: w \in F\right\}$.

Next we state the proposition

(21) If $f \in F$, then $f^{-1} \in F^{-1}$.

Let $F$ be an empty set. Note that $F^{-1}$ is empty.

Let $F$ be an extended real-membered non empty set. One can check that $F^{-1}$ is non empty.

The following propositions are true:

(22) If $F \subseteq G$, then $F^{-1} \subseteq G^{-1}$.

(23) $(F \cup G)^{-1}=F^{-1} \cup G^{-1}$.

(24) $(F \cap G)^{-1} \subseteq F^{-1} \cap G^{-1}$.

(25) $\ominus\left(F^{-1}\right)=(\ominus F)^{-1}$.

(26) $\{f\}^{-1}=\left\{f^{-1}\right\}$.

(27) $\{f, g\}^{-1}=\left\{f^{-1}, g^{-1}\right\}$.

Let $A$ be a complex-membered set. The functor $A^{-1}$ yields a complexmembered set and is defined as follows:

(Def. 4) $A^{-1}=\left\{c^{-1}: c \in A\right\}$.

Let us notice that the functor $A^{-1}$ is involutive.

One can prove the following propositions:

(28) $a \in A$ iff $a^{-1} \in A^{-1}$.

(29) $a^{-1} \in A$ iff $a \in A^{-1}$.

Let $A$ be an empty set. Observe that $A^{-1}$ is empty.

Let $A$ be a complex-membered non empty set. Observe that $A^{-1}$ is non empty.

Let $A$ be a real-membered set. Note that $A^{-1}$ is real-membered.

Let $A$ be a rational-membered set. One can verify that $A^{-1}$ is rationalmembered.

Let $A$ be a real-membered set and let $F$ be an extended real-membered set. One can verify that $A^{-1}$ and $F^{-1}$ can be identified when $A=F$.

Next we state several propositions:

(30) $A \subseteq B$ iff $A^{-1} \subseteq B^{-1}$.

(31) If $A^{-1}=B^{-1}$, then $A=B$.

(32) $(A \cup B)^{-1}=A^{-1} \cup B^{-1}$.

(33) $(A \cap B)^{-1}=A^{-1} \cap B^{-1}$.

(34) $(A \backslash B)^{-1}=A^{-1} \backslash B^{-1}$. 


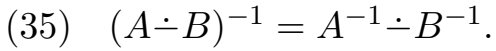

(36) $\ominus\left(A^{-1}\right)=(\ominus A)^{-1}$.

(37) $\{a\}^{-1}=\left\{a^{-1}\right\}$.

(38) $\{a, b\}^{-1}=\left\{a^{-1}, b^{-1}\right\}$.

Let $F, G$ be extended real-membered sets. The functor $F \oplus G$ is defined as follows:

(Def. 5) $F \oplus G=\left\{w_{1}+w_{2}: w_{1} \in F \wedge w_{2} \in G\right\}$.

Let us note that the functor $F \oplus G$ is commutative.

Next we state the proposition

(39) If $f \in F$ and $g \in G$, then $f+g \in F \oplus G$.

Let $F$ be an empty set and let $G$ be an extended real-membered set. Observe that $F \oplus G$ is empty and $G \oplus F$ is empty.

Let $F, G$ be extended real-membered non empty sets. One can check that $F \oplus G$ is non empty.

Let $F, G$ be extended real-membered sets. Observe that $F \oplus G$ is extended real-membered.

Next we state several propositions:

(40) If $F \subseteq G$ and $H \subseteq I$, then $F \oplus H \subseteq G \oplus I$.

(41) $F \oplus(G \cup H)=(F \oplus G) \cup(F \oplus H)$.

(42) $F \oplus G \cap H \subseteq(F \oplus G) \cap(F \oplus H)$.

(43) $\{f\} \oplus\{g\}=\{f+g\}$.

(44) $\{f\} \oplus\{g, h\}=\{f+g, f+h\}$.

(45) $\{f, g\} \oplus\{h, i\}=\{f+h, f+i, g+h, g+i\}$.

Let $A, B$ be complex-membered sets. The functor $A \oplus B$ is defined by:

(Def. 6) $A \oplus B=\left\{c_{1}+c_{2}: c_{1} \in A \wedge c_{2} \in B\right\}$.

Let us note that the functor $A \oplus B$ is commutative.

Next we state the proposition

(46) If $a \in A$ and $b \in B$, then $a+b \in A \oplus B$.

Let $A$ be an empty set and let $B$ be a complex-membered set. One can check that $A \oplus B$ is empty and $B \oplus A$ is empty.

Let $A, B$ be complex-membered non empty sets. Note that $A \oplus B$ is non empty.

Let $A, B$ be complex-membered sets. One can check that $A \oplus B$ is complexmembered.

Let $A, B$ be real-membered sets. Observe that $A \oplus B$ is real-membered.

Let $A, B$ be rational-membered sets. Observe that $A \oplus B$ is rational-membered.

Let $A, B$ be integer-membered sets. One can verify that $A \oplus B$ is integermembered.

Let $A, B$ be natural-membered sets. Observe that $A \oplus B$ is natural-membered. 
Let $A, B$ be real-membered sets and let $F, G$ be extended real-membered sets. Observe that $A \oplus B$ and $F \oplus G$ can be identified when $A=F$ and $B=G$.

We now state several propositions:

(47) If $A \subseteq B$ and $C \subseteq D$, then $A \oplus C \subseteq B \oplus D$.

(48) $A \oplus(B \cup C)=(A \oplus B) \cup(A \oplus C)$.

(49) $A \oplus B \cap C \subseteq(A \oplus B) \cap(A \oplus C)$.

(50) $(A \oplus B) \oplus C=A \oplus(B \oplus C)$.

(51) $\{a\} \oplus\{b\}=\{a+b\}$.

(52) $\{a\} \oplus\{s, t\}=\{a+s, a+t\}$.

(53) $\{a, b\} \oplus\{s, t\}=\{a+s, a+t, b+s, b+t\}$.

Let $F, G$ be extended real-membered sets. The functor $F \ominus G$ is defined by:

(Def. 7) $\quad F \ominus G=F \oplus \ominus G$.

Next we state two propositions:

(54) $F \ominus G=\left\{w_{1}-w_{2}: w_{1} \in F \wedge w_{2} \in G\right\}$.

(55) If $f \in F$ and $g \in G$, then $f-g \in F \ominus G$.

Let $F$ be an empty set and let $G$ be an extended real-membered set. Note that $F \ominus G$ is empty and $G \ominus F$ is empty.

Let $F, G$ be extended real-membered non empty sets. Observe that $F \ominus G$ is non empty.

Let $F, G$ be extended real-membered sets. Note that $F \ominus G$ is extended real-membered.

One can prove the following propositions:

(56) If $F \subseteq G$ and $H \subseteq I$, then $F \ominus H \subseteq G \ominus I$.

(57) $F \ominus(G \cup H)=(F \ominus G) \cup(F \ominus H)$.

(58) $F \ominus G \cap H \subseteq(F \ominus G) \cap(F \ominus H)$.

(59) $\ominus(F \oplus G)=\ominus F \ominus G$.

(60) $\ominus(F \ominus G)=\ominus F \oplus G$.

(61) $\{f\} \ominus\{g\}=\{f-g\}$.

(62) $\{f\} \ominus\{h, i\}=\{f-h, f-i\}$.

(63) $\{f, g\} \ominus\{h\}=\{f-h, g-h\}$.

(64) $\{f, g\} \ominus\{h, i\}=\{f-h, f-i, g-h, g-i\}$.

Let $A, B$ be complex-membered sets. The functor $A \ominus B$ is defined by:

(Def. 8) $\quad A \ominus B=A \oplus \ominus B$.

Next we state two propositions:

(65) $A \ominus B=\left\{c_{1}-c_{2}: c_{1} \in A \wedge c_{2} \in B\right\}$.

(66) If $a \in A$ and $b \in B$, then $a-b \in A \ominus B$.

Let $A$ be an empty set and let $B$ be a complex-membered set. One can check that $A \ominus B$ is empty and $B \ominus A$ is empty. 
Let $A, B$ be complex-membered non empty sets. One can verify that $A \ominus B$ is non empty.

Let $A, B$ be complex-membered sets. One can verify that $A \ominus B$ is complexmembered.

Let $A, B$ be real-membered sets. Note that $A \ominus B$ is real-membered.

Let $A, B$ be rational-membered sets. One can verify that $A \ominus B$ is rationalmembered.

Let $A, B$ be integer-membered sets. One can check that $A \ominus B$ is integermembered.

Let $A, B$ be real-membered sets and let $F, G$ be extended real-membered sets. One can check that $A \ominus B$ and $F \ominus G$ can be identified when $A=F$ and $B=G$.

The following propositions are true:

(67) If $A \subseteq B$ and $C \subseteq D$, then $A \ominus C \subseteq B \ominus D$.

(68) $A \ominus(B \cup C)=(A \ominus B) \cup(A \ominus C)$.

(69) $A \ominus B \cap C \subseteq(A \ominus B) \cap(A \ominus C)$.

(70) $\ominus(A \oplus B)=\ominus A \ominus B$.

(71) $\ominus(A \ominus B)=\ominus A \oplus B$.

(72) $A \oplus(B \ominus C)=(A \oplus B) \ominus C$.

(73) $A \ominus(B \oplus C)=A \ominus B \ominus C$.

(74) $\quad A \ominus(B \ominus C)=(A \ominus B) \oplus C$.

(75) $\{a\} \ominus\{b\}=\{a-b\}$.

(76) $\{a\} \ominus\{s, t\}=\{a-s, a-t\}$.

(77) $\{a, b\} \ominus\{s\}=\{a-s, b-s\}$.

(78) $\{a, b\} \ominus\{s, t\}=\{a-s, a-t, b-s, b-t\}$.

Let $F, G$ be extended real-membered sets. The functor $F \circ G$ is defined as follows:

(Def. 9) $\quad F \circ G=\left\{w_{1} \cdot w_{2}: w_{1} \in F \wedge w_{2} \in G\right\}$.

Let us observe that the functor $F \circ G$ is commutative.

Let $F$ be an empty set and let $G$ be an extended real-membered set. One can verify that $F \circ G$ is empty and $G \circ F$ is empty.

Let $F, G$ be extended real-membered sets. Note that $F \circ G$ is extended real-membered.

Next we state the proposition

(79) If $f \in F$ and $g \in G$, then $f \cdot g \in F \circ G$.

Let $F, G$ be extended real-membered non empty sets. Observe that $F \circ G$ is non empty.

One can prove the following propositions:

(80) $(F \circ G) \circ H=F \circ(G \circ H)$. 
(81) If $F \subseteq G$ and $H \subseteq I$, then $F \circ H \subseteq G \circ I$.

(82) $F \circ(G \cup H)=F \circ G \cup F \circ H$.

(83) $F \circ(G \cap H) \subseteq(F \circ G) \cap(F \circ H)$.

(84) $F \circ \ominus G=\ominus(F \circ G)$.

(85) $(F \circ G)^{-1}=F^{-1} \circ G^{-1}$.

(86) $\{f\} \circ\{g\}=\{f \cdot g\}$.

(87) $\{f\} \circ\{h, i\}=\{f \cdot h, f \cdot i\}$.

(88) $\{f, g\} \circ\{h, i\}=\{f \cdot h, f \cdot i, g \cdot h, g \cdot i\}$.

Let $A, B$ be complex-membered sets. The functor $A \circ B$ is defined as follows:

(Def. 10) $A \circ B=\left\{c_{1} \cdot c_{2}: c_{1} \in A \wedge c_{2} \in B\right\}$.

Let us notice that the functor $A \circ B$ is commutative.

One can prove the following proposition

(89) If $a \in A$ and $b \in B$, then $a \cdot b \in A \circ B$.

Let $A$ be an empty set and let $B$ be a complex-membered set. Note that $A \circ B$ is empty and $B \circ A$ is empty.

Let $A, B$ be complex-membered non empty sets. Note that $A \circ B$ is non empty.

Let $A, B$ be complex-membered sets. Note that $A \circ B$ is complex-membered.

Let $A, B$ be real-membered sets. Note that $A \circ B$ is real-membered.

Let $A, B$ be rational-membered sets. Observe that $A \circ B$ is rational-membered.

Let $A, B$ be integer-membered sets. Observe that $A \circ B$ is integer-membered.

Let $A, B$ be natural-membered sets. Observe that $A \circ B$ is natural-membered.

Let $A, B$ be real-membered sets and let $F, G$ be extended real-membered sets. Note that $A \circ B$ and $F \circ G$ can be identified when $A=F$ and $B=G$.

The following propositions are true:

(90) $(A \circ B) \circ C=A \circ(B \circ C)$.

(91) If $A \subseteq B$ and $C \subseteq D$, then $A \circ C \subseteq B \circ D$.

(92) $A \circ(B \cup C)=A \circ B \cup A \circ C$.

(93) $A \circ(B \cap C) \subseteq(A \circ B) \cap(A \circ C)$.

(94) $A \circ \ominus B=\ominus(A \circ B)$.

(95) $A \circ(B \oplus C) \subseteq A \circ B \oplus A \circ C$.

(96) $A \circ(B \ominus C) \subseteq A \circ B \ominus A \circ C$.

(97) $(A \circ B)^{-1}=A^{-1} \circ B^{-1}$.

(98) $\{a\} \circ\{b\}=\{a \cdot b\}$.

(99) $\{a\} \circ\{s, t\}=\{a \cdot s, a \cdot t\}$.

(100) $\{a, b\} \circ\{s, t\}=\{a \cdot s, a \cdot t, b \cdot s, b \cdot t\}$.

Let $F, G$ be extended real-membered sets. The functor $F \oslash G$ is defined as follows: 
(Def. 11) $F \oslash G=F \circ G^{-1}$.

We now state two propositions:

(101) $F \oslash G=\left\{\frac{w_{1}}{w_{2}}: w_{1} \in F \wedge w_{2} \in G\right\}$.

(102) If $f \in F$ and $g \in G$, then $\frac{f}{g} \in F \oslash G$.

Let $F$ be an empty set and let $G$ be an extended real-membered set. One can verify that $F \oslash G$ is empty and $G \oslash F$ is empty.

Let $F, G$ be extended real-membered non empty sets. One can verify that $F \oslash G$ is non empty.

Let $F, G$ be extended real-membered sets. One can verify that $F \oslash G$ is extended real-membered.

Next we state a number of propositions:

(103) If $F \subseteq G$ and $H \subseteq I$, then $F \oslash H \subseteq G \oslash I$.

(104) $(F \cup G) \oslash H=(F \oslash H) \cup(G \oslash H)$.

(105) $F \cap G \oslash H \subseteq(F \oslash H) \cap(G \oslash H)$.

(106) $F \oslash(G \cup H)=(F \oslash G) \cup(F \oslash H)$.

(107) $F \oslash G \cap H \subseteq(F \oslash G) \cap(F \oslash H)$.

(108) $F \circ G \oslash H=F \circ(G \oslash H)$.

(109) $(F \oslash G) \circ H=F \circ H \oslash G$.

(110) $F \oslash G \oslash H=F \oslash G \circ H$.

(111) $\{f\} \oslash\{g\}=\left\{\frac{f}{g}\right\}$.

(112) $\{f\} \oslash\{h, i\}=\left\{\frac{f}{h}, \frac{f}{i}\right\}$.

(113) $\{f, g\} \oslash\{h\}=\left\{\frac{f}{h}, \frac{g}{h}\right\}$.

(114) $\{f, g\} \oslash\{h, i\}=\left\{\frac{f}{h}, \frac{f}{i}, \frac{g}{h}, \frac{g}{i}\right\}$.

Let $A, B$ be complex-membered sets. The functor $A \oslash B$ is defined by:

(Def. 12) $A \oslash B=A \circ B^{-1}$.

We now state two propositions:

(115) $A \oslash B=\left\{\frac{c_{1}}{c_{2}}: c_{1} \in A \wedge c_{2} \in B\right\}$.

(116) If $a \in A$ and $b \in B$, then $\frac{a}{b} \in A \oslash B$.

Let $A$ be an empty set and let $B$ be a complex-membered set. One can check that $A \oslash B$ is empty and $B \oslash A$ is empty.

Let $A, B$ be complex-membered non empty sets. Note that $A \oslash B$ is non empty.

Let $A, B$ be complex-membered sets. Note that $A \oslash B$ is complex-membered.

Let $A, B$ be real-membered sets. Observe that $A \oslash B$ is real-membered.

Let $A, B$ be rational-membered sets. One can check that $A \oslash B$ is rationalmembered. 
Let $A, B$ be real-membered sets and let $F, G$ be extended real-membered sets. One can check that $A \oslash B$ and $F \oslash G$ can be identified when $A=F$ and $B=G$.

We now state a number of propositions:

(117) If $A \subseteq B$ and $C \subseteq D$, then $A \oslash C \subseteq B \oslash D$.

(118) $A \oslash(B \cup C)=(A \oslash B) \cup(A \oslash C)$.

(119) $A \oslash B \cap C \subseteq(A \oslash B) \cap(A \oslash C)$.

(120) $A \oslash \ominus B=\ominus(A \oslash B)$.

(121) $\ominus A \oslash B=\ominus(A \oslash B)$.

(122) $\quad(A \oplus B) \oslash C \subseteq(A \oslash C) \oplus(B \oslash C)$.

(123) $(A \ominus B) \oslash C \subseteq(A \oslash C) \ominus(B \oslash C)$.

(124) $A \circ B \oslash C=A \circ(B \oslash C)$.

(125) $(A \oslash B) \circ C=A \circ C \oslash B$.

(126) $A \oslash B \oslash C=A \oslash B \circ C$.

(127) $A \oslash(B \oslash C)=A \circ C \oslash B$.

(128) $\{a\} \oslash\{b\}=\left\{\frac{a}{b}\right\}$.

(129) $\{a\} \oslash\{s, t\}=\left\{\frac{a}{s}, \frac{a}{t}\right\}$.

(130) $\{a, b\} \oslash\{s\}=\left\{\frac{a}{s}, \frac{b}{s}\right\}$.

(131) $\{a, b\} \oslash\{s, t\}=\left\{\frac{a}{s}, \frac{a}{t}, \frac{b}{s}, \frac{b}{t}\right\}$.

Let $F$ be an extended real-membered set and let $f$ be an extended real number. The functor $f \oplus F$ is defined as follows:

(Def. 13) $f \oplus F=\{f\} \oplus F$.

We now state three propositions:

(132) If $g \in G$, then $f+g \in f \oplus G$.

(133) $f \oplus F=\{f+w: w \in F\}$.

(134) If $e \in f \oplus F$, then there exists $w$ such that $e=f+w$ and $w \in F$.

Let $F$ be an empty set and let $f$ be an extended real number. One can check that $f \oplus F$ is empty.

Let $F$ be an extended real-membered non empty set and let $f$ be an extended real number. Observe that $f \oplus F$ is non empty.

Let $F$ be an extended real-membered set and let $f$ be an extended real number. One can check that $f \oplus F$ is extended real-membered.

Next we state several propositions:

(135) If $r \oplus F \subseteq r \oplus G$, then $F \subseteq G$.

(136) If $r \oplus F=r \oplus G$, then $F=G$.

(137) $r \oplus F \cap G=(r \oplus F) \cap(r \oplus G)$.

(138) $(f \oplus F) \backslash(f \oplus G) \subseteq f \oplus(F \backslash G)$.

(139) $r \oplus(F \backslash G)=(r \oplus F) \backslash(r \oplus G)$. 
(140) $\quad r \oplus(F \dot{-} G)=(r \oplus F) \dot{-}(r \oplus G)$.

Let $A$ be a complex-membered set and let $a$ be a complex number. The functor $a \oplus A$ is defined as follows:

(Def. 14) $a \oplus A=\{a\} \oplus A$.

We now state three propositions:

(141) If $b \in A$, then $a+b \in a \oplus A$.

(142) $a \oplus A=\{a+c: c \in A\}$.

(143) If $e \in a \oplus A$, then there exists $c$ such that $e=a+c$ and $c \in A$.

Let $A$ be an empty set and let $a$ be a complex number. Observe that $a \oplus A$ is empty.

Let $A$ be a complex-membered non empty set and let $a$ be a complex number. Note that $a \oplus A$ is non empty.

Let $A$ be a complex-membered set and let $a$ be a complex number. Observe that $a \oplus A$ is complex-membered.

Let $A$ be a real-membered set and let $a$ be a real number. One can verify that $a \oplus A$ is real-membered.

Let $A$ be a rational-membered set and let $a$ be a rational number. Note that $a \oplus A$ is rational-membered.

Let $A$ be an integer-membered set and let $a$ be an integer number. One can verify that $a \oplus A$ is integer-membered.

Let $A$ be a natural-membered set and let $a$ be a natural number. Note that $a \oplus A$ is natural-membered.

Let $A$ be a real-membered set, let $F$ be an extended real-membered set, let $a$ be a real number, and let $f$ be an extended real number. Note that $a \oplus A$ and $f \oplus F$ can be identified when $a=f$ and $A=F$.

We now state several propositions:

(144) $A \subseteq B$ iff $a \oplus A \subseteq a \oplus B$.

(145) If $a \oplus A=a \oplus B$, then $A=B$.

(146) $0 \oplus A=A$.

(147) $(a+b) \oplus A=a \oplus(b \oplus A)$.

(148) $a \oplus(A \oplus B)=(a \oplus A) \oplus B$.

(149) $a \oplus A \cap B=(a \oplus A) \cap(a \oplus B)$.

(150) $a \oplus(A \backslash B)=(a \oplus A) \backslash(a \oplus B)$.

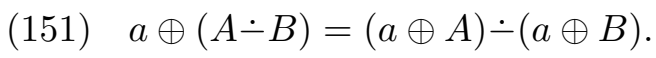

Let $F$ be an extended real-membered set and let $f$ be an extended real number. The functor $f \ominus F$ is defined by:

(Def. 15) $f \ominus F=\{f\} \ominus F$.

The following propositions are true:

(152) If $g \in G$, then $f-g \in f \ominus G$. 
(153) $f \ominus F=\{f-w: w \in F\}$.

(154) If $e \in f \ominus F$, then there exists $w$ such that $e=f-w$ and $w \in F$.

Let $F$ be an empty set and let $f$ be an extended real number. One can check that $f \ominus F$ is empty.

Let $F$ be an extended real-membered non empty set and let $f$ be an extended real number. One can verify that $f \ominus F$ is non empty.

Let $F$ be an extended real-membered set and let $f$ be an extended real number. Observe that $f \ominus F$ is extended real-membered.

We now state several propositions:

(155) If $r \ominus F \subseteq r \ominus G$, then $F \subseteq G$.

(156) If $r \ominus F=r \ominus G$, then $F=G$.

(157) $r \ominus F \cap G=(r \ominus F) \cap(r \ominus G)$.

(158) $r \ominus(F \backslash G)=(r \ominus F) \backslash(r \ominus G)$.

(159) $r \ominus(F \cdot G)=(r \ominus F) \dot{-}(r \ominus G)$.

Let $A$ be a complex-membered set and let $a$ be a complex number. The functor $a \ominus A$ is defined as follows:

(Def. 16) $a \ominus A=\{a\} \ominus A$.

Next we state three propositions:

(160) If $b \in A$, then $a-b \in a \ominus A$.

(161) $a \ominus A=\{a-c: c \in A\}$.

(162) If $e \in a \ominus A$, then there exists $c$ such that $e=a-c$ and $c \in A$.

Let $A$ be an empty set and let $a$ be a complex number. One can verify that $a \ominus A$ is empty.

Let $A$ be a complex-membered non empty set and let $a$ be a complex number. Note that $a \ominus A$ is non empty.

Let $A$ be a complex-membered set and let $a$ be a complex number. Note that $a \ominus A$ is complex-membered.

Let $A$ be a real-membered set and let $a$ be a real number. Note that $a \ominus A$ is real-membered.

Let $A$ be a rational-membered set and let $a$ be a rational number. Note that $a \ominus A$ is rational-membered.

Let $A$ be an integer-membered set and let $a$ be an integer number. Observe that $a \ominus A$ is integer-membered.

Let $A$ be a real-membered set, let $F$ be an extended real-membered set, let $a$ be a real number, and let $f$ be an extended real number. Observe that $a \ominus A$ and $f \ominus F$ can be identified when $a=f$ and $A=F$.

Next we state several propositions:

(163) $A \subseteq B$ iff $a \ominus A \subseteq a \ominus B$.

(164) If $a \ominus A=a \ominus B$, then $A=B$. 
(165) $\quad a \ominus A \cap B=(a \ominus A) \cap(a \ominus B)$.

(166) $\quad a \ominus(A \backslash B)=(a \ominus A) \backslash(a \ominus B)$.

(167) $a \ominus(A \dot{-} B)=(a \ominus A) \dot{-}(a \ominus B)$.

Let $F$ be an extended real-membered set and let $f$ be an extended real number. The functor $F \ominus f$ is defined as follows:

(Def. 17) $F \ominus f=F \ominus\{f\}$.

One can prove the following three propositions:

(168) If $g \in G$, then $g-f \in G \ominus f$.

(169) $F \ominus f=\{w-f: w \in F\}$.

(170) If $e \in F \ominus f$, then there exists $w$ such that $e=w-f$ and $w \in F$.

Let $F$ be an empty set and let $f$ be an extended real number. One can verify that $F \ominus f$ is empty.

Let $F$ be an extended real-membered non empty set and let $f$ be an extended real number. Observe that $F \ominus f$ is non empty.

Let $F$ be an extended real-membered set and let $f$ be an extended real number. Note that $F \ominus f$ is extended real-membered.

One can prove the following propositions:

(171) $\quad F \ominus f=\ominus(f \ominus F)$.

(172) $f \ominus F=\ominus(F \ominus f)$.

(173) $F \cap G \ominus r=(F \ominus r) \cap(G \ominus r)$.

(174) $(F \backslash G) \ominus r=(F \ominus r) \backslash(G \ominus r)$.

(175) $(F \dot{-} G) \ominus r=(F \ominus r) \dot{-}(G \ominus r)$.

Let $A$ be a complex-membered set and let $a$ be a complex number. The functor $A \ominus a$ is defined by:

(Def. 18) $A \ominus a=A \ominus\{a\}$.

Next we state three propositions:

(176) If $b \in A$, then $b-a \in A \ominus a$.

(177) $A \ominus a=\{c-a: c \in A\}$.

(178) If $e \in A \ominus a$, then there exists $c$ such that $e=c-a$ and $c \in A$.

Let $A$ be an empty set and let $a$ be a complex number. Observe that $A \ominus a$ is empty.

Let $A$ be a complex-membered non empty set and let $a$ be a complex number. Observe that $A \ominus a$ is non empty.

Let $A$ be a complex-membered set and let $a$ be a complex number. Observe that $A \ominus a$ is complex-membered.

Let $A$ be a real-membered set and let $a$ be a real number. Note that $A \ominus a$ is real-membered.

Let $A$ be a rational-membered set and let $a$ be a rational number. Note that $A \ominus a$ is rational-membered. 
Let $A$ be an integer-membered set and let $a$ be an integer number. One can verify that $A \ominus a$ is integer-membered.

Let $A$ be a real-membered set, let $F$ be an extended real-membered set, let $a$ be a real number, and let $f$ be an extended real number. One can verify that $A \ominus a$ and $F \ominus f$ can be identified when $a=f$ and $A=F$.

Next we state several propositions:

(179) $A \subseteq B$ iff $A \ominus a \subseteq B \ominus a$.

(180) If $A \ominus a=B \ominus a$, then $A=B$.

(181) $A \ominus a=\ominus(a \ominus A)$.

(182) $a \ominus A=\ominus(A \ominus a)$.

(183) $A \cap B \ominus a=(A \ominus a) \cap(B \ominus a)$.

(184) $(A \backslash B) \ominus a=(A \ominus a) \backslash(B \ominus a)$.

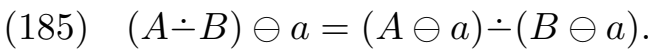

Let $F$ be an extended real-membered set and let $f$ be an extended real number. The functor $f \circ F$ is defined as follows:

(Def. 19) $f \circ F=\{f\} \circ F$.

The following three propositions are true:

(186) If $g \in G$, then $f \cdot g \in f \circ G$.

(187) $f \circ F=\{f \cdot w: w \in F\}$.

(188) If $e \in f \circ F$, then there exists $w$ such that $e=f \cdot w$ and $w \in F$.

Let $F$ be an empty set and let $f$ be an extended real number. Observe that $f \circ F$ is empty.

Let $F$ be an extended real-membered non empty set and let $f$ be an extended real number. One can verify that $f \circ F$ is non empty.

Let $F$ be an extended real-membered set and let $f$ be an extended real number. Note that $f \circ F$ is extended real-membered.

One can prove the following four propositions:

(189) If $r \neq 0$, then $r \circ(F \cap G)=(r \circ F) \cap(r \circ G)$.

(190) $f \circ F \backslash f \circ G \subseteq f \circ(F \backslash G)$.

(191) If $r \neq 0$, then $r \circ(F \backslash G)=r \circ F \backslash r \circ G$.

(192) If $r \neq 0$, then $r \circ(F \dot{\circ} G)=r \circ F \dot{-} r \circ G$.

Let $A$ be a complex-membered set and let $a$ be a complex number. The functor $a \circ A$ is defined as follows:

(Def. 20) $a \circ A=\{a\} \circ A$.

We now state three propositions:

(193) If $b \in A$, then $a \cdot b \in a \circ A$.

(194) $a \circ A=\{a \cdot c: c \in A\}$.

(195) If $e \in a \circ A$, then there exists $c$ such that $e=a \cdot c$ and $c \in A$. 
Let $A$ be an empty set and let $a$ be a complex number. Note that $a \circ A$ is empty.

Let $A$ be a complex-membered non empty set and let $a$ be a complex number. One can verify that $a \circ A$ is non empty.

Let $A$ be a complex-membered set and let $a$ be a complex number. Note that $a \circ A$ is complex-membered.

Let $A$ be a real-membered set and let $a$ be a real number. Note that $a \circ A$ is real-membered.

Let $A$ be a rational-membered set and let $a$ be a rational number. Observe that $a \circ A$ is rational-membered.

Let $A$ be an integer-membered set and let $a$ be an integer number. Note that $a \circ A$ is integer-membered.

Let $A$ be a natural-membered set and let $a$ be a natural number. One can check that $a \circ A$ is natural-membered.

Let $A$ be a real-membered set, let $F$ be an extended real-membered set, let $a$ be a real number, and let $f$ be an extended real number. Note that $a \circ A$ and $f \circ F$ can be identified when $a=f$ and $A=F$.

One can prove the following propositions:

(196) If $a \neq 0$ and $a \circ A \subseteq a \circ B$, then $A \subseteq B$.

(197) If $a \neq 0$ and $a \circ A=a \circ B$, then $A=B$.

(198) If $a \neq 0$, then $a \circ(A \cap B)=(a \circ A) \cap(a \circ B)$.

(199) If $a \neq 0$, then $a \circ(A \backslash B)=a \circ A \backslash a \circ B$.

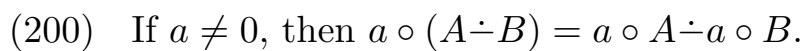

(201) $0 \circ A \subseteq\{0\}$.

(202) If $A \neq \emptyset$, then $0 \circ A=\{0\}$.

(203) $1 \circ A=A$.

(204) $(a \cdot b) \circ A=a \circ(b \circ A)$.

(205) $\quad a \circ(A \circ B)=(a \circ A) \circ B$.

(206) $\quad(a+b) \circ A \subseteq a \circ A \oplus b \circ A$.

(207) $\quad(a-b) \circ A \subseteq a \circ A \ominus b \circ A$.

(208) $a \circ(B \oplus C)=a \circ B \oplus a \circ C$.

(209) $a \circ(B \ominus C)=a \circ B \ominus a \circ C$.

Let $F$ be an extended real-membered set and let $f$ be an extended real number. The functor $f \oslash F$ is defined by:

(Def. 21) $f \oslash F=\{f\} \oslash F$.

We now state three propositions:

(210) If $g \in G$, then $\frac{f}{g} \in f \oslash G$.

(211) $f \oslash F=\left\{\frac{f}{w}: w \in F\right\}$.

(212) If $e \in f \oslash F$, then there exists $w$ such that $e=\frac{f}{w}$ and $w \in F$. 
Let $F$ be an empty set and let $f$ be an extended real number. Note that $f \oslash F$ is empty.

Let $F$ be an extended real-membered non empty set and let $f$ be an extended real number. One can verify that $f \oslash F$ is non empty.

Let $F$ be an extended real-membered set and let $f$ be an extended real number. Observe that $f \oslash F$ is extended real-membered.

Let $A$ be a complex-membered set and let $a$ be a complex number. The functor $a \oslash A$ is defined by:

(Def. 22) $a \oslash A=\{a\} \oslash A$.

One can prove the following three propositions:

(213) If $b \in A$, then $\frac{a}{b} \in a \oslash A$.

(214) $a \oslash A=\left\{\frac{a}{c}: c \in A\right\}$.

(215) If $e \in a \oslash A$, then there exists $c$ such that $e=\frac{a}{c}$ and $c \in A$.

Let $A$ be an empty set and let $a$ be a complex number. One can check that $a \oslash A$ is empty.

Let $A$ be a complex-membered non empty set and let $a$ be a complex number. Note that $a \oslash A$ is non empty.

Let $A$ be a complex-membered set and let $a$ be a complex number. One can check that $a \oslash A$ is complex-membered.

Let $A$ be a real-membered set and let $a$ be a real number. One can check that $a \oslash A$ is real-membered.

Let $A$ be a rational-membered set and let $a$ be a rational number. One can verify that $a \oslash A$ is rational-membered.

Let $A$ be a real-membered set, let $F$ be an extended real-membered set, let $a$ be a real number, and let $f$ be an extended real number. Observe that $a \oslash A$ and $f \oslash F$ can be identified when $a=f$ and $A=F$.

The following propositions are true:

(216) If $a \neq 0$ and $a \oslash A \subseteq a \oslash B$, then $A \subseteq B$.

(217) If $a \neq 0$ and $a \oslash A=a \oslash B$, then $A=B$.

(218) If $a \neq 0$, then $a \oslash A \cap B=(a \oslash A) \cap(a \oslash B)$.

(219) If $a \neq 0$, then $a \oslash(A \backslash B)=(a \oslash A) \backslash(a \oslash B)$.

(220) If $a \neq 0$, then $a \oslash(A-B)=(a \oslash A) \div(a \oslash B)$.

(221) $\quad(a+b) \oslash A \subseteq(a \oslash A) \oplus(b \oslash A)$.

(222) $(a-b) \oslash A \subseteq(a \oslash A) \ominus(b \oslash A)$.

Let $F$ be an extended real-membered set and let $f$ be an extended real number. The functor $F \oslash f$ is defined by:

(Def. 23) $F \oslash f=F \oslash\{f\}$.

We now state three propositions:

(223) If $g \in G$, then $\frac{g}{f} \in G \oslash f$. 
(224) $F \oslash f=\left\{\frac{w}{f}: w \in F\right\}$.

(225) If $e \in F \oslash f$, then there exists $w$ such that $e=\frac{w}{f}$ and $w \in F$.

Let $F$ be an empty set and let $f$ be an extended real number. Note that $F \oslash f$ is empty.

Let $F$ be an extended real-membered non empty set and let $f$ be an extended real number. Observe that $F \oslash f$ is non empty.

Let $F$ be an extended real-membered set and let $f$ be an extended real number. Note that $F \oslash f$ is extended real-membered.

Let $A$ be a complex-membered set and let $a$ be a complex number. The functor $A \oslash a$ is defined by:

(Def. 24) $A \oslash a=A \oslash\{a\}$.

One can prove the following three propositions:

(226) If $b \in A$, then $\frac{b}{a} \in A \oslash a$.

(227) $A \oslash a=\left\{\frac{c}{a}: c \in A\right\}$.

(228) If $e \in A \oslash a$, then there exists $c$ such that $e=\frac{c}{a}$ and $c \in A$.

Let $A$ be an empty set and let $a$ be a complex number. Note that $A \oslash a$ is empty.

Let $A$ be a complex-membered non empty set and let $a$ be a complex number. Note that $A \oslash a$ is non empty.

Let $A$ be a complex-membered set and let $a$ be a complex number. One can check that $A \oslash a$ is complex-membered.

Let $A$ be a real-membered set and let $a$ be a real number. One can check that $A \oslash a$ is real-membered.

Let $A$ be a rational-membered set and let $a$ be a rational number. Observe that $A \oslash a$ is rational-membered.

Let $A$ be a real-membered set, let $F$ be an extended real-membered set, let $a$ be a real number, and let $f$ be an extended real number. Note that $A \oslash a$ and $F \oslash f$ can be identified when $a=f$ and $A=F$.

The following propositions are true:

(229) If $a \neq 0$ and $A \oslash a \subseteq B \oslash a$, then $A \subseteq B$.

(230) If $a \neq 0$ and $A \oslash a=B \oslash a$, then $A=B$.

(231) If $a \neq 0$, then $A \cap B \oslash a=(A \oslash a) \cap(B \oslash a)$.

(232) If $a \neq 0$, then $(A \backslash B) \oslash a=(A \oslash a) \backslash(B \oslash a)$.

(233) If $a \neq 0$, then $(A \dot{-} B) \oslash a=(A \oslash a) \dot{-}(B \oslash a)$.

(234) $\quad(A \oplus B) \oslash a=(A \oslash a) \oplus(B \oslash a)$.

(235) $\quad(A \ominus B) \oslash a=(A \oslash a) \ominus(B \oslash a)$. 


\section{REFERENCES}

[1] Grzegorz Bancerek. The ordinal numbers. Formalized Mathematics, 1(1):91-96, 1990.

[2] Andrzej Trybulec. Enumerated sets. Formalized Mathematics, 1(1):25-34, 1990.

[3] Andrzej Trybulec. On the sets inhabited by numbers. Formalized Mathematics, 11(4):341$347,2003$.

[4] Zinaida Trybulec. Properties of subsets. Formalized Mathematics, 1(1):67-71, 1990.

Received December 19, 2008 